\title{
Applicability of Sigmoid Colon Graft for Vaginal Replacement (Colovaginoplasty) at Young Age
}

\author{
Mohamed A. Baky Fahmy ${ }^{1}$, Hanaa Abdel Hamid M. Al Abeissy ${ }^{1}$, Mohamed M. Abdalla ${ }^{2}$ \\ ${ }^{1}$ Al Azher University, Cairo, Egypt \\ ${ }^{2}$ Al Galaa Teaching Hospital, Cairo, Egypt \\ E-mail: ymabfahmy@yahoo.com
}

Received June 9, 2011; revised August 13, 2011; accepted August 25, 2011

\begin{abstract}
Objective: to evaluate the applicability, feasibility, complications, short and long term results of vaginoplasty by using sigmoid colon graft with stapler assistance for resuming colonic continuity in children with absent vagina. Patients and Methods: records of 15 patients 8 of them had Androgen Insensitivity Syndrome, 4 had congenital vaginal atresia, and 3 had Congenital Adrenal Hyperplasia, underwent sigmoid graft vaginal reconstruction with the aid of circular stapler during the period from 2005 to 2010 were reviewed retrospectively. Results: a neovagina was constructed in those patients who aged from 3 to 9 years (mean 4 years), where sigmoid colon was used in all patients. Hospital stay ranged from 5 to 12 days (mean 7), operative time ranged from $60 \mathrm{~min}$ to $120 \mathrm{~min}$ (mean $75 \mathrm{~min}$ ). All patients were followed up 6 monthly in the first year and then yearly, complications occurred in 4 patients: introital stenosis in 2, and another 2 cases had minor wound infection. The need for neovaginal dilation was indicated in 5 out of 15 patients. There was no complications related to bowel anastomosis, no mortality in this group and the cosmetic results were excellent in all cases. Conclusion: sigmoid colovaginoplasty appears to be applicable for remedy of vaginal atresia at any age. It is associated with a less complication rate, the long term results is satisfactory and it seems a better technique, especially in girls who doesn't expect sexual activity shortly after surgery.
\end{abstract}

Keywords: Vaginal Replacement, Vaginal Atresia, Stapler, Neovagina, Colovaginoplasty and Sigmoid Graft

\section{Introduction}

Surgical management of girls with congenital absent vagina continues to be a major technical problem due to a prospective functional and psychological effect of the outcome. The majority of cases of absent vagina result from abnormality results from congenital adrenal hypertrophy, müllerian duct failure, androgen insensitivity syndrome and gonadal dysgenesis [1]. Construction of an artificial vagina had undergone a long evolution from conservative to various surgical methods since 1938 when Frank proposed a nonsurgical approach using various instruments and saddle-like devices that exerted pressure on the vaginal vestibule [2]. This method replaced the technique proposed by Baldwin in 1904 [3], whereby the vagina was reconstructed using an isolated segment of small intestine, Pratt in 1961 proposed the use of sigmoid colon in vaginal reconstruction [4]. With the same purpose in mind cecum had been used, others proposed a dermatome-collected cutaneous graft, mu- cous membrane flap harvested from the bladder, and peritoneal fragment advanced from the Douglas pouch. Vaginal reconstruction was also performed with skin island flaps created in the perineal and inguinal region [7]. It is widely accepted that staplers use for resuming bowel anastomosis is effective in reducing operating time and potential complications related to their use specially in the pediatric population [8]. In this study the sigmoid graft used for vaginal reconstruction winning the advantage of surgical stapler to reduce the time of resuming bowel continuity and to minimize the complication rate related to bowel anastomosis and the whole procedure.

\section{Patients and Method}

During a period of 5 years, from 2005 to 2010 a total of 15 patients had an indication for vaginal replacement due to different pathology was reviewed.

Abdominal ultrasound, chromosomal analysis and hor- 
monal assay were performed preoperatively for all patients, Magnetic Resonance Imaging (MRI) and ascending cystourtherogram done for 10 patients to assess the associated anomalies and the status of the existing genital organs. Patients with AIS (8 patients) underwent gonadectomy before vaginoplasty and patients who had $\mathrm{CAH}$ (3 patients) underwent clitoral surgery either along the vaginoplasty, or latter on (Figures 1 and 2).

\section{Technique}

The surgical procedure for creating a sigmoid colon neovagina was done basically according to the principles described by Graziano et al. [9] with some modifications mainly in establishing bowel continuity by stapler. Patients were admitted to the hospital 2 days before the surgery for mechanical and antibiotic bowel preparation. Through a small abdominal Pfannenstiel incision or an existing abdominal scar, the descending and sigmoid colon were mobilized. The mesentery was examined to

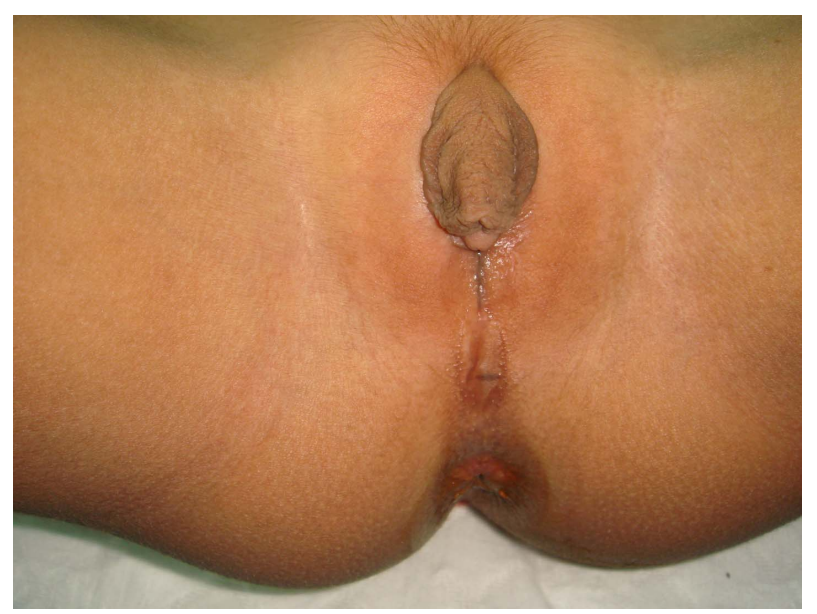

Figure 1. A case of adrenogenital hyperplasia.

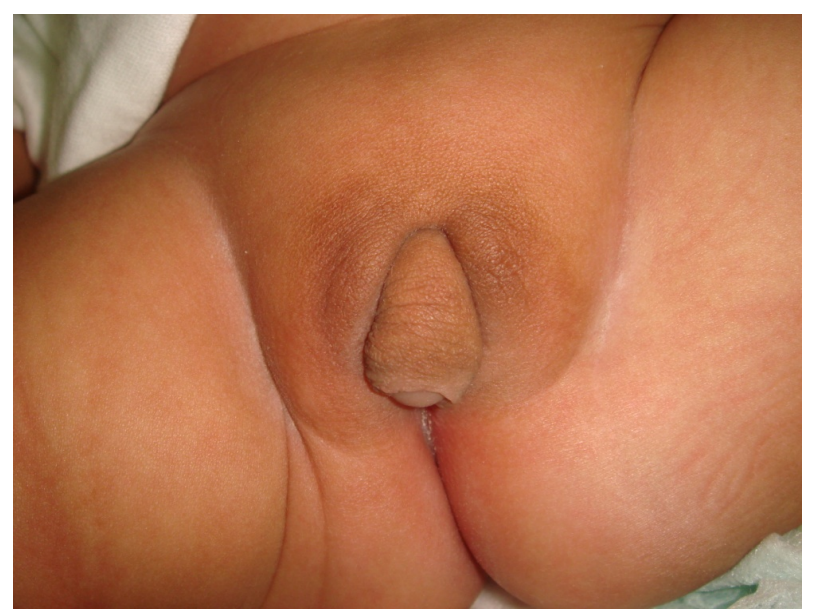

Figure 2. A case of androgen insensitivity syndrome. identify vascularisation and to evaluate the length of the sigmoid loop which will be used for vaginal construction. A $10 \mathrm{~cm}$ to $12 \mathrm{~cm}$ segment of the sigmoid colon containing at least 2 sigmoid arteries was isolated on its vascular pedicle for transposition to the perineum, and the mesenteric defect was closed.

Depending on the individual patient's vascular anatomy, either an iso-peristaltic or anti-peristaltic orientation was used. The proximal end of the sigmoid segment was closed with interrupted absorbable sutures and fixed to the vestigial remnants of the Mullerian ducts or to the uterosacral ligaments. The continuity of the colon was restored by an appropriate size (from $21 \mathrm{~mm}$ to $28 \mathrm{~mm}$ ) curved disposable intraluminal gastrointestinal anastomosis (GIA) stapler, and we preferred the Curved Detachable Head (CDH) type (By Ethicon Endo-Surgery, one of Johnson \& Johnson Company) where the handle introduced from the anus, with minimal anal dilation to join the head of stapler at the proximal bowel (Figure 3).

We performed the abdominal procedures first, and after the preparation of the sigmoid loop, the upper side of the rectovesical space was opened and enlarged to contain the distal end of the sigmoid loop, in cases of vaginal atresia a surgical plane was created between the urethra, bladder and rectum by sharp dissection, but in GAH cases the sigmoid graft connected to the vaginal remnant. The mobilized sigmoid loop was brought down to the perineal canal without tension to create a colon-perineal anastomosis with interrupted absorbable sutures. To prevent early retraction, the lateral portions of the sigmoid neovagina were also fixed to the levator ani muscles with interrupted absorbable sutures. Finally, the neovagina was packed with Povidone-iodine soaked gauze, which was removed 24 hours after surgery, and an indwelling Foley's catheter was left in place for 2 to 3 days. At discharge from the hospital patients were instructed to irrigate the neovagina daily for 3 weeks and weekly there-

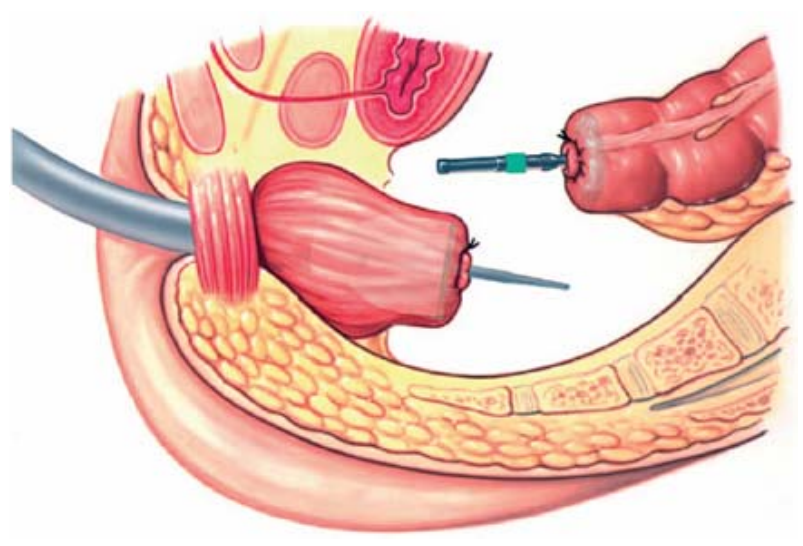

Figure 3. Curved disposable intraluminal GIA stapler for bowel. 
after with Povidone-iodine solution. Calibration of the neovagina done 2 weeks after discharge and during the follow up visits, and if there is any need for dilatation it is scheduled two weekly under local anesthetic. All patients had been followed up from 6 months to 4 years in six months intervals for the first year and then yearly.

\section{Results}

A total of 15 girls aged from aged from 1 to 8 years (mean 3), 8 of them had Androgen Insensitivity Syndrome (AIS), 4 had congenital vaginal atresia, and 3 had CAH (Figures 4 and 5).

Operative time ranged from $60 \mathrm{~min}$ to $120 \mathrm{~min}$ (mean 75). Hospital stay ranged from 5 to 12 days means 7 days. All patients were followed up from 6 month to 4 years (mean 2 years), and calibration done 2 weeks after discharge and during the follow up visits, by an appropriate size Hegar's dilators, the need for neovaginal dilation was evaluated and it was indicated in only 5 out of 15 patients for 4 sessions in 5 and for 6 sessions in 3 patients with local anesthetic. Excessive mucous discharge observed in 6 patients (older one) but it abstained gradually with the neovaginal wash by Povidone-iodine solution.

Complications occurred in 4 patients, introital stenosis in 2 patients, one of them lost during the follow up due to

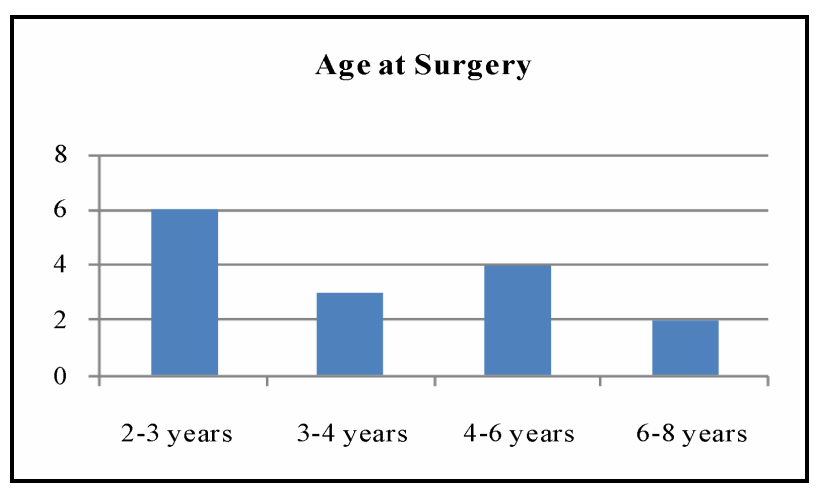

Figure 4. Age distribution at surgery.

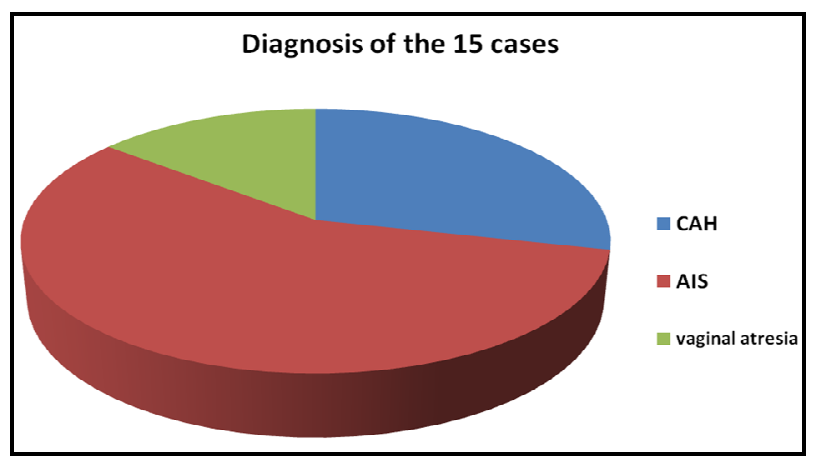

Figure 5. Cases distribution. some social problems but came back into sight with tight stenosis which necessitates redo of the introital wound, and 2 cases had minor wound infection which managed conservatively without any surgical intervention and without sequels. There was no complications related to bowel anastomosis, and there is no mortality in this group of patients. All patients had functional adequate patency and lubricant neovagina, with good patient satisfaction and the vagina had good appearing introits, mucus production decreased 3 to 4 month after the operation (Figure 6).

As the tradition in our society that the girls had no sexual activity outside marriage, so it seems difficult to assess the sexual function of the reconstructed vagina, which dictates a further follow up to evaluate the sexual activity and functional effectiveness of the neovagina latter on.

\section{Discussion}

Vaginal reconstruction may be indicated in cases of Mayer-Rokitansky-Kuster-Hauser Syndrome [5], where the vagina is congenitally absent, intersex and for other cases of Androgen insensitivity Syndrome. Reconstructing the vagina in the children and young adults may be a great technical challenge. Patients were more likely to present after puberty with primary amenorrhea or failure of intercourse, but recently the gender assignment to a neonate with ambiguous genitalia became crucial [7]. Most investigators, however, suggest timing for surgery from the newborn to 3-year-old period. Reasons for this early intervention include better compliance with dilations, lessening of the parents concerns regarding their "anomalous" child, and the assumption that the child later in life does not remember early interventions [6-7].

Numerous surgical and non-surgical methods have been proposed to create a vaginal tunnel, the aim of any

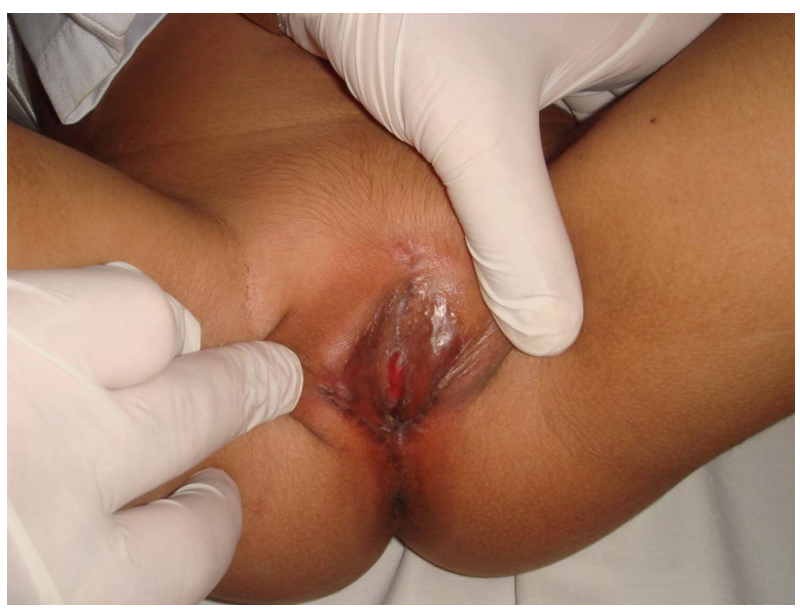

Figure 6. Postoperative. 
method is to provide a canal of adequate length, which is lined with mucous membrane, characterised by a low tendency to stenosis and comfortable during sexual activity. Although used as a first-line of treatment for decades, skin grafts and dilation techniques are associated with a high incidence of shrinkage, lack of lubrication and dyspareunia [10].

Intestinal transplant methods to create a functioning neovagina were advocated more than 100 years ago; the advantages of these methods include adequate vaginal length, natural lubrication, early intercourse and a low rate of shrinkage [3]. Most segments of the intestinal tract have been used to create a neovagina, but the sigmoid colon is particularly useful because it is anatomically close to the perineum, it is sufficiently long and the mobility of its vascular pedicle allows it to be brought into the perineum [11].

Also the bowel vaginoplasty utilizing sigmoid colon offers some advantages over skin grafts because of distensibility and self lubricating property with no tendency for neovagina stenosis, but it has the drawbacks of a laparotomy with visible scars, possibility of bowel leakage/obstruction and problem of mucous secretion [12]. Also the sigmoid segments of the colon could be liable for inflammatory bowel disease [13], primary adenocarcinoma has been reported, so vaginoscopy should be used for any case of postoperative bleeding, pain, or suspected mass .There have been reports of mucosal prolapse, as well as of stenosis [14].

The Vecchietti technique [15] and its laparoscopic variant requires a vaginal dimple of $3-4 \mathrm{~cm}$, a long time and rigorous cooperation by the patients to achieve acceptable results [15].

In the last decades, due to the progress in anesthesia, antibiotic use and the reduction of risks associated with colorectal anastomosis, the sigmoid graft technique has became the first-line choice for the treatment of vaginal agenesis in both children and adults [16]. A sigmoid neovagina is cosmetically pleasing, offers natural lubrication and during creation can be anchored to prevent prolapse.

A collective analysis of 202 patients from 7 recent series of bowel neovaginoplasty found a complication rate of $35 \%$ and a re-operation rate of $4 \%$. These rates of complications are not comparable to those reported for skin graft vaginoplasties [10].

In this study we used the sigmoid graft as a preferable procedure in 15 patients aged from 1 to 8 years (mean 3 years), a historical cohort study of those consecutive patients who underwent neovagina reconstruction were presented.

A dedicated database was reviewed for the etiology of vaginal malformation, surgical complications and post- operative follow-up, where Androgen Insensitivity Syndrome (AIS) was the common cause of vaginal atresia (8 patients), 4 had congenital vaginal atresia (Figure 5).

In this small series the introduction of surgical stapler for resuming the bowel continuity enabled to shorten the time of surgery (60 min to $120 \mathrm{~min}$, mean 75), to reduce the hospital stay which ranged from 5 to 12 days means 7 days, and we claim that it is also limits the complications of bowel anastomosis to nil.

But other complications encountered in 4 patients, introital stenosis in 2 patients, one of them had a tight stenosis which necessitates redo of the introital wound, and 2 cases had minor postoperative wound infection which managed conservatively with local wash and systemic antibiotic for 5 days without any surgical intervention and without sequels. There were no long term complications or impediment related to bowel anastomosis, and there is no mortality in this group of patients. All patients had functional adequate patency and lubricant neovagina, with good patient satisfaction and the vagina had fine appearing introits and mucus production decreased 3 to 4 month after the operation.

\section{Conclusions}

Vagina could be reconstructed with better success rate and low complication rate by using sigmoid colon, it does not require moulds, dilatation, lubricant, and the vagina grows with the child and could be used in different diagnosed vaginal atresia, especially in girls who doesn't expect sexual activity shortly after surgery. The use of stapler to reestablish the bowel continuity seems to minimize the time of the technique and decline the rate of complications related to bowel anastomosis. The long-term satisfaction with the sigmoid neovagina for intercourse, especially in girls had their vagina constructed before puberty, still requires long-term evaluation.

\section{References}

[1] D. M. Powell, K. D. Newman and J. Randloph, “A Proposed Classification of Vaginal Anomalies and Their Surgical Correction,” Journal of Pediatric Surgery, Vol. 30, No. 2, 1995, pp. 271-276. doi:10.1016/0022-3468(95)90573-1

[2] R. T. Frank, "The Formation of an Artificial Vagina without Operation,” American Journal of Obstetrics \& Gynecology, Vol. 35, 1938, pp. 1053-1055.

[3] J. F. Baldwin, "The Formation of an Artificial Vagina by Intestinal Transplantation,” Annals of Surgery, Vol. 40, No. 3, 1904, pp. 398-403.

[4] J. H. Pratt, "Sigmoidovaginostomy: A New Method of Obtaining Satisfactory Vaginal Depth,” American Jour- 
nal of Obstetrics \& Gynecology, Vol. 81, No. 3, 1961, pp. 535-545.

[5] G. E. Griffin, C. Edwards, J. M. Madden, M. J. Harrod, and J. D. Wilson, "Congenital Absence of the Vagina the Mayer-Rokitansky-Kuster-Hauser Syndrome," Annals of Internal Medicine, Vol. 85, No. 2, 1976, pp. 2224-2236.

[6] R. J. Miller and L. L. Breech, "Surgical Correction of Vaginal Anomalies," Clinical Obstetrics and Gynecology, Vol. 51, No. 2, 2008, pp. 223-236. doi:10.1097/GRF.0b013e31816d2181

[7] J. M. Schober, "Long-Term Outcomes and Changing Attitudes to Intersexuality,” BJU International, Vol. 83, No. S3, 1999, pp. 39-50. doi:10.1046/j.1464-410x.1999.0830s3039.x

[8] G. Mattioli, M. Castagnetti, P. Repetto, et al., "Complications of Mechanical Suturing in Pediatric Patients," Journal of Pediatric Surgery, Vol. 38, No. 7, 2003, pp. 1051- 1054. doi:10.1016/S0022-3468(03)00190-8

[9] K. Graziano, D. H. Teitelbaum, R. B. Hirschl and A. G. Coran, "Vaginal Reconstruction for Ambiguous Genitalia and Congenital Absence of the Vagina: A 27-Year Experience," Journal of Pediatric Surgery, Vol. 37, No. 7, 2002, pp. 955- 960. doi:10.1053/jpsu.2002.33815

[10] A. Rajimwale, P. D. Furness, W. O. Brant and M. A. Koyle, "Vaginal Construction Using Sigmoid Colon in Children and Young Adults,” BJU International, Vol. 94,
No. 1, 2004, pp. 115-119. doi:10.1111/j.1464-4096.2004.04911.x

[11] W. H. Hendren and A. Atala, "Use of Bowel for Vaginal Reconstruction,” The Journal of Urology, Vol. 152, No. 2, 1997, pp. 752-755.

[12] R. Lenaghan, N. Wilson, C. E. Lucas and A. M. Ledgerwood, "The Role of Rectosigmoid Neocolporrhaphy," Surgery, Vol. 122, No. 4, 1997, pp. 856-860. doi:10.1016/S0039-6060(97)90098-2

[13] D. P. Froese, R. Haggitt and W. G. Friend, "Ulcerative Colitis in Autotransplanted Neovagina," Gastroenterology, Vol. 100, No. 6, 1991, pp. 1749-1752.

[14] M. Urrsic-Vrscaj, J. Lindtner, J. Lavomovec et al., "Adenocarcinoma in Sigmoid Neovagina," European Journal of Gyneacological/Oncology, Vol. 15, No. 1, 1994, pp. 24-28.

[15] F. Borruto, S. T. Chasen, F. A. Chervenak and L. Fedele, "The Vecchietti Procedure for Surgical Treatment of Vaginal Agenesis: Comparison of Laparoscopy and Laparotomy,” International Journal of Gynaecology and Obstetrics, Vol. 64, No. 2, 1999, pp. 153-158. doi:10.1016/S0020-7292(98)00244-6

[16] J. C. Goligher, “The Use of Pedicled Transplant of Sigmoid for Vaginal Construction," Annals of the Royal College of Surgeons of England, Vol. 65, No. 6, 1983, pp. 353-355.

\section{Appendix}

\section{Abbreviation}

Androgen Insensitivity Syndrome (AIS), Congenital Adrenal Hyperplasia (CAH), Gastrointestinal Anastomosis (GIA), Curved Detachable Head (CDH). 